\title{
OSCILLATION CRITERIA FOR SELF-ADJOINT DIFFERENTIAL SYSTEMS
}

\author{
BY \\ WILLIAM T. REID( $(1)$
}

1. Introduction. For a differential system with complex coefficients that is of the general form of the accessory differential equations for a calculus of variations problem of Lagrange or Bolza type that is identically normal (see, for example, Bliss $[4, \S 81]$, Morse $[11 ; 12]$, Reid $[15 ; 16 ; 18 ; 20])$, variational methods are employed to establish certain oscillation criteria involving associated two-point boundary problems.

In particular, application of these general results to special systems which are equivalent to self-adjoint scalar quasi-differential equations of even order yields corresponding criteria for these equations. It is to be emphasized that for such scalar equations the definitions of conjugate point and oscillation employed here are the restrictive ones arising from the corresponding concepts for the associated differential system, and lack the generality corresponding to the definitions introduced by Leighton and Nehari [10] for real fourth order equations

$$
\left(r(x) u^{\prime \prime}\right)^{\prime \prime}-p(x) u=0 .
$$

In view of Theorem 3.6 of [10], however, a very special instance of one of the criteria developed here provides a variational proof of the recently established result of $\mathrm{H}$. Howard [7, Theorem 2.1], to the effect that if $r(x)$ and $p(x)$ are real-valued positive functions with $r(x) \in C^{\prime \prime}(0, \infty), p(x) \in C(0, \infty)$, and for $a>0$ we have $\int_{a}^{\infty}[r(x)]^{-1} d x=\infty$, then (1.1) is nonoscillatory on $(a, \infty)$ if and only if for $b>a$ the smallest proper value $\lambda_{b}$ of the boundary problem

$$
\left(r(x) u^{\prime \prime}\right)^{\prime \prime}-\lambda p(x) u=0, u=u^{\prime}=0 \text { at } x=a, r u^{\prime \prime}=\left(r u^{\prime \prime}\right)^{\prime}=0 \text { at } x=b
$$

satisfies $\lambda_{b}>1$. The criteria here derived also include as special cases some of the results of Barrett [2].

The formulation of the general system under consideration is given in $\$ 2$, together with the statement of the basic results of the spectral theory for allied boundary problems, and the ensuing proof of a comparison theorem whose result is basic for the specific oscillation criteria of \$3. In connection with the general criterion of Theorem 3.2 it is to be noted that one of the

Presented to the Society, January 25, 1961; received by the editors January 21, 1961.

(1) This research was sponsored by the Mathematical Sciences Directorate, Air Force Office of Scientific Research of the Air Research and Development Command, under Contract No. AF 49(638)-994. 
basic assumptions involves the concept of a principal solution as developed recently by Reid [20]. Finally, $\$ 4$ is devoted to the application of the general results to self-adjoint scalar quasi-differential equations of even order as cited above.

Matrix notation is used throughout; in particular, matrices of one column are termed vectors, and for a vector $y=\left(y_{\alpha}\right),(\alpha=1, \cdots, n)$, the norm $|y|$ is given by $\left(\left|y_{1}\right|^{2}+\cdots+\left|y_{n}\right|^{2}\right)^{1 / 2}$. The $n \times n$ identity matrix is denoted by $E_{n}$, or merely $E$ when there is no ambiguity, and 0 is used indiscriminately for the zero matrix of any dimensions; the conjugate transpose of a matrix $M$ is designated by $M^{*}$. The relations $M \geqq N,(M>N)$, are used to signify that $M$ and $N$ are hermitian matrices of the same dimensions and $M-N$ is a non-negative (positive), definite matrix. If the elements of a matrix $M(x)$ are a.c. (absolutely continuous) on an interval $[c, d]$, then $M^{\prime}(x)$ signifies the matrix of derivatives at values for which these derivatives exist and the zero matrix elsewhere; correspondingly, if the elements of $M(x)$ are (Lebesgue) integrable on $[c, d]$ then $\int_{c}^{d} M(x) d x$ denotes the matrix of integrals of respective elements of $M(x)$. If matrices $M(x)$ and $N(x)$ are equal a.e. (almost everywhere) on their domain of definition we write simply $M(x)=N(x)$. In the totality of finite dimensional rectangular matrices with elements defined on a given interval $[c, d]$ we denote by $\mathbb{R}[c, d]$ the set of all matrices whose elements are (Lebesgue) integrable on $[c, d]$, and by $\ell_{2}[c, d]$ the set of all matrices $M$ whose elements $M_{\alpha \beta}(x)$ are measurable and $\left|M_{\alpha \beta}(x)\right|^{2} \in \mathbb{R}[c, d]$. For brevity, a matrix is termed continuous, etc., when each element of the matrix possesses the specified property.

2. A general self-adjoint differential system. For $x$ on a given ray $X: a<x<\infty$ of the real line, let $\omega(x, y, \pi)$ denote the hermitian form

$$
\omega(x, y, \pi) \equiv \pi^{*} R(x) \pi+\pi^{*} Q(x) y+y^{*} Q^{*}(x) \pi+y^{*} P(x) y
$$

in the $2 n$ variables $(y, \pi)=\left(y_{1}, \cdots, y_{n}, \pi_{1}, \cdots, \pi_{n}\right)$ with $R(x), Q(x), P(x)$ $n \times n$ matrices having complex-valued continuous elements on $X$, and $R(x)$, $P(x)$ hermitian on this interval. In addition, consider a vector linear form

$$
\Phi(x, y, \pi) \equiv \phi(x) \pi+\theta(x) y,
$$

where $\phi(x)$ and $\theta(x)$ are $m \times n(m<n)$ matrices with complex continuous elements on $X$, and satisfying the following hypothesis:

$\left(H_{1}\right)$ For $x \in X$ the matrix $\phi(x)$ is of rank $m$, and $\pi^{*} R(x) \pi>0$ for arbitrary non-null vectors $\pi$ satisfying $\phi(x) \pi=0$.

In particular, $\left(\boldsymbol{H}_{1}\right)$ implies that the $(m+n) \times(m+n)$ hermitian matrix

$$
\left\|\begin{array}{cc}
R(x) & \phi^{*}(x) \\
\phi(x) & 0
\end{array}\right\|
$$

is nonsingular on $X$. 
For the Bolza type variational problem involving the functional

$$
\Im[y ; c, d]=\int_{c}^{d} \omega\left(x, y(x), y^{\prime}(x)\right) d x,
$$

subject to the auxiliary $m$-dimensional vector differential equation

$$
\Phi\left(x, y(x), y^{\prime}(x)\right)=0,
$$

the Euler-Lagrange differential equations in vector form become

$$
\begin{gathered}
{\left[R(x) u^{\prime}+Q(x) u+\phi^{*}(x) \mu\right]^{\prime}-\left[Q^{*}(x) u^{\prime}+P(x) u+\theta^{*}(x) \mu\right]=0,} \\
\Phi\left(x, u, u^{\prime}\right)=0,
\end{gathered}
$$

where $u(x)$ is an $n$-dimensional vector function and $\mu(x)$ is an $m$-dimensional "multiplier" vector function.

As in Reid [17, §4], if the inverse of $(2.3)$ is written as

$$
\left\|\begin{array}{cc}
T(x) & \tau^{*}(x) \\
\tau(x) & t(x)
\end{array}\right\|
$$

where $T(x)$ and $t(x)$ are hermitian matrices of respective orders $n$ and $m$, and $\tau(x)$ is an $m \times n$ matrix, then in terms of the canonical variables

$$
u(x), \quad v(x)=R(x) u^{\prime}(x)+Q(x) u(x)+\phi^{*}(x) \mu(x)
$$

the equations (2.5) may be written as the first order differential system

$$
u^{\prime}=A(x) u+B(x) v, \quad v^{\prime}=C(x) u-A^{*}(x) v,
$$

with the coefficient matrices

$$
A=-\left(T Q+\tau^{*} \theta\right), \quad B=T, \quad C=P-Q^{*} T Q-Q^{*} \tau^{*} \theta-\theta^{*} \tau Q-\theta^{*} t \theta .
$$

Moreover, the matrices $B$ and $C$ of $(2.8)$ are hermitian on $X$, and $B$ is a nonnegative definite matrix of rank $n-m$ with $B \phi^{*} \equiv 0$.

In [18] the author has discussed various oscillation criteria for a vector differential system (2.7) equivalent to the Euler equation for a functional (2.4) with no auxiliary differential restraints $\Phi=0$, and with the coefficient matrices $A, B, C$ satisfying weaker conditions than those imposed above. For the system under present consideration the conditions may be relaxed in a corresponding fashion, but for simplicity attention is restricted to the case described above.

Throughout the following discussion we shall make the following assumption on (2.5), or equivalently on the canonical system (2.7).

$\left(H_{2}\right)$ The system is identically normal on $X$; that is, if $X_{1}$ is an arbitrary nondegenerate subinterval of $X$, and $u(x) \equiv 0, v(x)$ is a solution of (2.7) on $X_{1}$, then $v(x) \equiv 0$ on $X_{1}$.

For $[c, d]$ a compact subinterval of $X$ the symbol $\mathfrak{D}[c, d]=\mathfrak{D}_{* *}[c, d]$ will 
signify the class of vector functions $y(x)$ which are differentially admissible on $[c, d]$ in the sense that on this subinterval $y(x)$ is a.c., $y^{\prime}(x) \in \mathbb{R}_{2}[c, d]$, while $\Phi\left(x, y(x), y^{\prime}(x)\right)=0$ on $[c, d]$. Because of the frequent occurrence of certain linear subclasses of $\mathfrak{D}[c, d]$ we introduce the further notation: $\mathfrak{D}_{0 *}[c, d]$ $=\{y \mid y \in \mathfrak{D}[c, d], y(c)=0\}, \mathfrak{D}_{* 0}[c, d]=\{y \mid y \in \mathfrak{D}[c, d], y(d)=0\}, \mathfrak{D}_{00}[c, d]$ $=\{y \mid y \in \mathfrak{D}[c, d], y(c)=0=y(d)\}$.

We shall be concerned with the four boundary problems consisting of the linear vector differential system

$$
u^{\prime}=A(x) u+B(x) v, \quad v^{\prime}=C(x) u-A^{*}(x) v-\lambda K(x) u,
$$

in the characteristic parameter $\lambda$, and the two-point boundary conditions

$$
\begin{array}{ll}
\Delta_{* *}[c, d]: v(c)=0, v(d)=0 ; & \Delta_{0 *}[c, d]: u(c)=0, v(d)=0 ; \\
\Delta_{* 0}[c, d]: v(c)=0, u(d)=0 ; & \Delta_{00}[c, d]: u(c)=0, u(d)=0 .
\end{array}
$$

For brevity, these boundary problems will be referred to as $\mathfrak{B}_{* *}[c, d]$, $\mathfrak{B}_{0 *}[c, d], \mathfrak{B}_{* 0}[c, d]$, and $\mathfrak{B}_{00}[c, d]$, respectively. Throughout the following discussion it will be assumed that $K(x)$ is a hermitian matrix which is continuous on $X$, and $\Re[y, w ; c, d]$ will signify the hermitian functional

$$
\Re[y, w ; c, d]=\int_{0}^{d} w^{*}(x) K(x) y(x) d x
$$

for $y(x), w(x)$ vector functions of $\mathfrak{R}_{2}[c, d]$; for brevity, we set $\mathfrak{R}[y ; c, d]$ $=\Omega[y, y ; c, d]$. For the special case of $K(x) \equiv E$ the functionals $\int_{c}^{d} w^{*}(x) y(x) d x$ and $\int_{c}^{d} y^{*}(x) y(x) d x$ are denoted by $\mathbb{E}[y, w ; c, d]$ and $\mathbb{E}[y ; c, d]$, respectively. We shall be concerned primarily with matrices $K(x)$ possessing the following property on compact subintervals $[c, d]$ of $X$, where $(p, q)$ may denote any one of the sets $(*, *),(*, 0),(0, *)$, or $(0,0)$.

$P_{p q}[c, d]: K(x)$ is a continuous hermitian matrix such that $\Omega[y ; c, d]$ is positive definite on $\mathfrak{D}_{p q}[c, d]$, that is, $\Re[y ; c, d]>0$ for arbitrary $y(x) \in \mathfrak{D}_{p q}[c, d]$ with $y(x) \not \equiv$ on $[c, d]$.

The basic results of the spectral theory for the above defined boundary problems are given in the following theorem.

THEOREM 2.1. If hypotheses $\left(H_{1}\right)$ and $\left(H_{2}\right)$ hold for $(2.7),[c, d] \subset X$, and for $(p, q)$ either $(*, *),(*, 0),(0, *)$, or $(0,0)$ the matrix $K(x)$ has property $P_{p q}[c, d]$, then $\mathfrak{V}_{p q}[c, d]$ possesses a sequence of proper values $\lambda_{j}^{p q}=\lambda_{j}^{p q}[c, d]$, $(j=1,2, \cdots)$, and corresponding proper solutions $u=u_{j}^{p q}(x)=u_{j}^{p q}(x ; c, d)$ of (2.9) for $\lambda=\lambda_{j}^{p o}$ such that:

(i) $\lambda_{j}^{p q} \leqq \lambda_{j+1}^{p q},(j=1,2, \cdots)$, and $\lambda_{j}^{p o} \rightarrow \infty$ as $j \rightarrow \infty$;

(ii) $\Im\left[u_{j}^{p p} ; c, d\right]=\lambda_{j}^{p q}, \Omega\left[u_{j}^{p q}, u_{k}^{p q} ; c, d\right]=\delta_{j k},(j, k=1,2, \cdots)$;

(iii) $\Im[y ; c, d] \geqq \lambda_{j}^{p q}$ for $y \in\left\{y \mid y \in \mathcal{D}_{p q}[c, d], \Re[y ; c, d]=1, \Re\left[y, u_{\mathbf{k}}^{p q} c c, d\right]\right.$ $=0$ for $k<j\}$, and the equality sign holds only if there is a corresponding vector function $z(x)$ such that $u=y(x), v=z(x)$ is a solution of (2.9) for $\lambda=\lambda_{j}^{x}$. 
It is to be remarked that the above definition of differential admissibility is more general than the one frequently used, which restricts the functions to have piecewise continuous derivatives. In references to previous literature no distinction will be made in regard to the class of differentially admissible functions employed by the cited author, however, since the corresponding results for the wider class of admissible functions may be established by obvious modifications, or are readily derivable from the stated results by wellknown approximation theorems for the Lebesgue integral.

In the case of real differential systems (2.9) with $K(x) \equiv E$, the results of Theorem 2.1 were first established by Morse $[11 ; 12]$ in his extensions of the classical Sturmian theory to general accessory boundary problems associated with identically normal Lagrange variational problems; an alternate treatment of such problems is given by $\mathrm{Hu}$ [8]. If $K(x)$ is positive definite and the coefficient matrices of $(2.9)$ are real-valued on $[c, d]$, then the results of Theorem 2.1 are explicitly given by Reid $[15 ; 16]$; moreover, for such real systems the general results are contained in either [15] or [16] as soon as the following result is established.

Lemma 2.1. If hypothesis $\left(H_{1}\right)$ holds and $\Omega[y ; c, d]$ is positive definite on $\mathfrak{D}_{p q}[c, d]$, then there exists a constant $l=l_{p q}[c, d]$ such that $\Im[y ; c, d \mid l] \equiv \Im[y ; c, d]$ $+l \Re[y ; c, d]$ is positive definite on $\mathfrak{D}_{p q}[c, d]$.

Indeed, if the result of this lemma is not true then for $j=1,2, \ldots$ there exists a vector function $y_{j}(x) \in \mathfrak{D}_{p q}[c, d]$ such that $\Im\left[y_{j} ; c, d \mid j\right] \leqq 0, \mathfrak{E}\left[y_{j} ; c, d\right]$ $=1$. Now (see, for example [16, Lemma 3.2]), there exist positive constants $m_{0}, l_{0}$ such that $\Im[y ; c, d] \geqq m_{0} \mathbb{E}\left[y^{\prime} ; c, d\right]-l_{0} \mathbb{E}[y ; c, d]$ for $y \in \mathfrak{D}[c, d]$. Consequently,

$$
m_{0} \mathbb{E}\left[y_{j}^{\prime} ; c, d\right]+j \Omega\left[y_{j} ; c, d\right] \leqq l_{0}, \quad \mathbb{E}\left[y_{j} ; c, d\right]=1 \quad(j=1,2, \cdots),
$$

so that $\mathbb{E}\left[y_{j}^{\prime} ; c, d\right] \leqq l_{0} / m_{0}(j=1,2, \cdots)$ and $\Re\left[y_{j} ; c, d\right] \rightarrow 0$ as $j \rightarrow \infty$. In view of the boundedness of the sequences $\left\{\mathbb{E}\left[y_{j} ; c, d\right]\right\},\left\{\mathbb{E}\left[y_{j}^{\prime} ; c, d\right]\right\}$ the vector functions $y_{j}(x)$ are uniformly bounded and equicontinuous on $[c, d]$, and by classical function theory results there exists a $y(x) \in \mathfrak{D}_{p q}[c, d]$ and a subsequence $\left\{y_{j_{k}}(x)\right\}$ such that $y_{j_{k}}(x) \rightarrow y(x)$ uniformly on $[c, d]$, while the sequence $\left\{y_{j k}^{\prime}(x)\right\}$ converges weakly to $y^{\prime}(x)$ in the Hilbert space $\mathfrak{l}_{2}[c, d]$, so that we have $\mathbb{E}[y ; c, d]=1, \Re[y ; c, d]=0$, contrary to the assumption that $\Re[y ; c, d]$ is positive definite on $\mathfrak{D}_{p q}[c, d]$.

For the above problems $\mathfrak{B}_{p q}[c, d]$ with complex coefficients, the results of Theorem 2.1 may be obtained from the corresponding results for an associated problem with real coefficients involving $2 n$-dimensional real-valued vector functions, or by methods entirely analogous to those employed in the case of real coefficients; in this latter regard, the reader is referred to Reid $[18 ; 20]$. In particular, once Lemma 2.1 is established the methods of Morse [11; 12; 13, Ch. IV], Birkhoff and Hestenes [3], Hestenes [5], Hu [8], or Reid [15; 
$16 ; 18 ; 20$ ] may be used to establish the general results of Theorem 2.1. In particular, the following results are ready consequences of the extremizing properties of the proper values of the boundary problems $\mathfrak{B}_{p q}[c, d]$ stated in Theorem 2.1.

COROLLARY 1. If $m$ is the smallest integer such that $\lambda_{m}^{p q}[c, d] \geqq 0$, then $m-1$ is the negative index of $\Im[y ; c, d]$ on $\mathfrak{D}_{p q}[c, d]$, that is, $m-1$ is the dimension of a maximal linear subclass of $\mathfrak{D}_{p q}[c, d]$ on which $\Im[y ; c, d]$ is negative definite; in particular, $m$ is independent of the matrix $K(x)$ satisfying $P_{p a}[c, d]$ and occurring in the boundary problem $\mathfrak{B}_{p e}[c, d]$.

Corollary 2. $\mathfrak{B}_{00}[c, d]$ is a subproblem of dimension $n$ of each of the problems $\mathfrak{B}_{0 *}[c, d]$ and $\mathfrak{B}_{* 0}[c, d]$, while $\mathfrak{B}_{0 *}[c, d], \mathfrak{B}_{* 0}[c, d]$ are individually subproblems of dimension $n$ of $\mathfrak{B}_{* *}[c, d]$, and consequently for $j=1,2, \cdots$,

$$
\begin{array}{ll}
\lambda_{j}^{0 *}[c, d] \leqq \lambda_{j}^{00}[c, d] \leqq \lambda_{j+n}^{0 *}[c, d], & \lambda_{j}^{* 0}[c, d] \leqq \lambda_{j}^{00}[c, d] \leqq \lambda_{j+n}^{* 0}[c, d], \\
\lambda_{j}^{* *}[c, d] \leqq \lambda_{j}^{0 *}[c, d] \leqq \lambda_{j+n}^{* *}[c, d], & \lambda_{j}^{* *}[c, d] \leqq \lambda_{j}^{* 0}[c, d] \leqq \lambda_{j+*}^{* *}[c, d] .
\end{array}
$$

In particular, a ready consequence of Theorem 2.1 is the following comparison theorem, which is basic for the oscillation criteria of the next section.

ThEOREM 2.2. If $b, c, d$ are points of $X$ satisfying $b\langle c\langle d$, and $r+s\rangle n$, then $\lambda_{r+t-n}^{00}[b, d] \leqq \operatorname{Max}\left(\lambda_{r}^{0 *}[b, c], \lambda_{\imath}^{* 0}[c, d]\right)$.

Indeed, since $r+s>n$ there exist constants $e_{1}, \cdots, e_{r}, f_{1}, \cdots, f_{\text {o }}$ such that the vector function $y(x)$ defined by

$$
y(x)=\sum_{j=1}^{r} u_{j}^{0 *}(x ; b, c) e_{j} \text { on }[b, c], \quad y(x)=\sum_{k=1}^{\dot{1}} u_{k}^{* 0}(x ; c, d) f_{k} \text { on }(c, d],
$$

is continuous on $[b, d]$, satisfies with $u_{n}^{00}=u_{n}^{00}(x ; b, d)$ the $r+s-n-1$ orthogonality conditions $\Omega\left[y, u_{h}^{00} ; b, d\right]=0$ for $h<r+s-n$, while $\Omega[y ; b, d]$ $=\left|e_{1}\right|^{2}+\cdots+\left|e_{r}\right|^{2}+\left|f_{1}\right|^{2}+\cdots+\left|f_{s}\right|^{2}=1$. Then $y(x) \in D_{00}[b, d]$, and by Theorem 2.1 we have

$$
\begin{aligned}
\lambda_{r+d-n}^{\infty 0}[b, d] \leqq \Im[y ; b, d] & =\sum_{j=1}^{r} \lambda_{j}^{0 *}[b, c]\left|e_{j}\right|^{2}+\sum_{k=1}^{\dot{ }} \lambda_{k}^{* 0}[c, d]\left|f_{k}\right|^{2}, \\
& \leqq \lambda_{r}^{0 *}[b, c] \sum_{j=1}^{r}\left|e_{j}\right|^{2}+\lambda_{*}^{* 0}[c, d] \sum_{k=1}^{*}\left|f_{k}\right|^{2}, \\
& \leqq M\left[\sum_{j=1}^{r}\left|e_{j}\right|^{2}+\sum_{k=1}^{\dot{1}}\left|f_{k}\right|^{2}\right]=M,
\end{aligned}
$$

where $M=\operatorname{Max}\left(\lambda_{r}^{0 *}[b, c], \lambda_{s}^{* 0}[c, d]\right)$. 
Finally, in view of the form of the boundary problems considered by Nehari [14], Leighton and Nehari [10], and Howard [7], we note the following result.

TheOREM 2.3. Suppose that hypotheses $\left(H_{1}\right)$ and $\left(H_{2}\right)$ hold, while for a compact subinterval $[c, d]$ of $X$ and $(p, q)$ one of the sets $(0,0),(0, *),(*, 0),(*, *)$, we have $C(x)=C_{1}(x)-C_{2}(x)$, where $C_{1}(x), C_{2}(x)$ are continuous hermitian matrices with $C_{2}(x)$ having property $P_{p q}[c, d]$. If $\tilde{B}_{p q}[c, d]$ denotes the boundary problem consisting of the differential system

$$
u^{\prime}=A(x) u+B(x) v, \quad v^{\prime}=C_{1}(x) u-A^{*}(x) v-\lambda C_{2}(x) u,
$$

with the corresponding boundary conditions $\Delta_{p q}[c, d]$, and $\lambda=\lambda_{j}^{p}[c, d]$ $(j=1,2, \cdots)$ are the proper values of $\tilde{\mathfrak{B}}_{p q}[c, d]$ as specified by Theorem 2.1, then for $j=1,2, \ldots$ we have $\lambda_{j}^{p e}[c, d] \leqq 1$ if and only if $\lambda_{j}^{\mu}[c, d] \leqq 0$ for the corresponding boundary problem $\mathfrak{B}_{p \mathrm{q}}[c, d]$ involving (2.9) with $K(x)$ any hermitian matrix having property $P_{p q}[c, d]$.

The result of Theorem 2.3 is an immediate consequence of Corollary 1 to Theorem 2.1 , and the fact that for $K(x) \equiv C_{2}(x)$ the boundary problem $\tilde{\mathfrak{B}}_{p q}[c, d]$ is equivalent to $\mathfrak{B}_{p q}[c, d]$ under the linear change of parameter $\lambda=\lambda+1$.

3. Oscillation criteria. A point $x_{2}$ is said to be conjugate to $x_{1}$, (with respect to the differential system (2.7)), if there exists a nontrivial solution $u(x)$, $v(x)$ of this system with $u\left(x_{1}\right)=0=u\left(x_{2}\right)$. The system is termed nonoscillatory on a given interval if no two distinct points of this interval are mutually conjugate.

Now in view of hypothesis $\left(H_{1}\right)$ a necessary and sufficient condition for (2.7) to be nonoscillatory on a compact subinterval $\left[x_{1}, x_{2}\right]$ of $X$ is that $\Im\left[y ; x_{1}, x_{2}\right]$ be positive definite on $\mathfrak{B}_{00}\left[x_{1}, x_{2}\right]$. The reader is referred to Bliss $[4, \S \S 89-91]$, or Morse [12, Part III], for a proof in the case of systems with real coefficients, and the details are readily extensible to systems with complex coefficients. In particular, for systems (2.7) satisfying $\left(H_{1}\right),\left(H_{2}\right)$ one may employ the methods used in Reid [18, Theorem 2.1] to establish the corresponding result for similar systems involving no auxiliary differential equations $\Phi\left(x, y, y^{\prime}\right)=0$.

Throughout this section it will be assumed that hypotheses $\left(H_{1}\right),\left(H_{2}\right)$ hold, and that $K(x)$ satisfies $P_{* *}[c, d]$ for arbitrary nondegenerate compact subintervals $[c, d]$ of $X$. In view of the extremizing property of the smallest proper value of the boundary problem $\mathfrak{B}_{00}[c, d]$, it then follows that (2.7) is nonoscillatory on a given subinterval $X_{0}$ of $X$ if and only if $\lambda_{1}^{00}[c, d]>0$ for arbitrary $[c, d] \subset X_{0}$. In particular, from Corollary 2 of Theorem 2.1 it follows that a sufficient condition for nonoscillation on $X_{0}$ is $\lambda_{1}^{0 *}[c, d]>0$ for arbitrary $[c, d] \subset X_{0}$, although in general this latter condition is not necessary 
for nonoscillation. In certain circumstances this latter condition is also necessary, however, as is shown by the following theorem.

THEOREM 3.1. Suppose that for arbitrary $b \in X$ there exists $a d>b$ such that $\lambda_{n}^{* 0}[b, d]<0$. Then for a given $x_{1} \in X$ a necessary and sufficient condition for (2.7) to be nonoscillatory on $\left[x_{1}, \infty\right)$ is that $\lambda_{1}^{0 *}\left[x_{1}, x_{2}\right]>0$ for all $x_{2}>x_{1}$.

In view of the remark preceding the statement of the theorem, only a proof of the necessity of the condition is required. Now if $x_{1} \in X$ and there exists a $b>x_{1}$ such that $\lambda_{1}^{0 *}\left[x_{1}, b\right] \leqq 0$, let $d>b$ be a value such that $\lambda_{n}^{* 0}[b, d] \leqq 0$ as assured by the hypothesis of the theorem. From Theorem 2.2 it then follows that $\lambda_{1}^{\nu^{0}}\left[x_{1}, d\right] \leqq 0$, and consequently $(2.7)$ is oscillatory on $\left[x_{1}, d\right]$.

From the extremizing properties of the proper values $\lambda_{j}^{* 0}[b, d]$ it follows that for a fixed value $b \in X$ the $j$ th proper value $\lambda_{j}^{* 0}[b, d]$ is a monotone decreasing function of $d$ on $(b, \infty)$ (see, for example, Morse [12, Part III] or Reid $[16,86])$ and consequently the assumption that there exists a $d>b$ such that $\lambda_{0}^{* 0}[b, d]<0$ is no stronger than the condition that there exists a $d_{1}>b$ such that $\lambda_{n}^{* 0}\left[b, d_{1}\right] \leqq 0$.

In view of Corollary 2 to Theorem 2.1 , the condition $\lambda_{n}^{* 0}[b, d]<0$ implies that $\lambda_{n}^{* *}[b, d]<0$, although in general the converse is not true. We shall proceed to show, however, that under certain circumstances the hypothesis of Theorem 3.1 may be replaced by the condition that for arbitrary $b \in X$ there exists a $d>b$ such that $\lambda_{n}^{* *}[b, d]<0$.

Let $\left(H_{3}\right)$ denote the following hypothesis:

$\left(H_{3}\right)$ If $c \in X$ there exists a continuous hermitian matrix $C_{0}(x)$ such that for arbitrary $d>c$ the functional $\int_{c}^{d} y^{*}(x)\left[C_{0}(x)-C(x)\right] y(x) d x$ is non-negative on $\mathfrak{D}_{* 0}[c, d]$, and a constant hermitian matrix $M \geqq 0$ such that if $(U ; V)$ $=\left(U_{0}(x) ; V_{0}(x)\right)$ is the solution of the matrix differential system

$$
U^{\prime}=A(x) U+B(x) V, \quad V^{\prime}=C_{0}(x) U-A^{*}(x) V,
$$

satisfying $U_{0}(x)=E, V_{0}(c)=M$, then $U_{0}(x)$ is nonsingular on $[c, \infty)$, and

$$
S\left(x, c ; U_{0}\right)=\int_{0}^{x} U_{0}^{-1}(t) B(t) U_{0}^{*-1}(t) d t
$$

is such that $\pi^{*} S\left(x, c ; U_{0}\right) \pi \rightarrow \infty$ as $x \rightarrow \infty$ for arbitrary non-null constant vectors $\pi$.

In the terminology of Reid $[18 ; 20],\left(U_{0}(x) ; V_{0}(x)\right)$ is a matrix of conjoined solutions of (3.1), that is, $U_{0}^{*} V_{0}-V_{0}^{*} U_{0} \equiv 0$. For $x \geqq c$ the matrix $S\left(x, c ; U_{0}\right)$ of $(3.2)$ is clearly hermitian and non-negative, and hence the positive definiteness of this matrix for $x>c$ is assured if it is shown that it is nonsingular for such values of $x$. Now, (see Reid $[20, \S 3]$ ), if

$$
U_{1}(x)=U_{0}(x) S\left(x, c ; U_{0}\right), \quad V_{1}(x)=V_{0}(x) S\left(x, c ; U_{0}\right)+U_{0}^{*-1}(x),
$$

then $(U ; V)=\left(U_{1}(x) ; V_{1}(x)\right)$ is the matrix of conjoined solutions of (3.1) 
satisfying the initial conditions $U_{1}(c)=0, V_{1}(c)=E$. If $x_{2}>c$, and $\xi$ is a vector such that $S\left(x_{2}, c ; U_{0}\right) \xi=0$, then since clearly $0 \leqq S\left(x, c ; U_{0}\right) \leqq S\left(x_{2}, c ; U_{0}\right)$ for $c \leqq x \leqq x_{2}$ it follows that $S\left(x, c ; U_{0}\right) \xi \equiv 0$ on $\left[c, x_{2}\right]$. Then $u(x)=U_{1}(x) \xi$, $v(x)=V_{1}(x) \xi$ is a solution of the differential system $u^{\prime}=A(x) u+B(x) v$, $v^{\prime}=C_{0}(x) u-A^{*}(x) v$ with $u \equiv 0$ on $\left[c, x_{2}\right]$, and hence $u(x), v(x)$ is also a solution of (2.7) with $u \equiv 0$ on $\left[c, x_{2}\right]$, so that by the normality hypothesis $\left(H_{2}\right)$ it follows that $0 \equiv v \equiv V_{1}(x) \xi$ on $\left[c, x_{2}\right]$. In particular, $0=V_{1}(c) \xi=\xi$, thus establishing that for $x>c$ the matrix $S\left(x, c ; U_{0}\right)$ is nonsingular, and consequently also positive definite.

If $\rho=\rho_{1}(x)$ is the smallest proper value of the matrix $S\left(x, c ; \dot{U}_{0}\right)$ then the monotone character of $S\left(x, c ; U_{0}\right)$ implies that $\rho_{1}(x)$ is monotone nondecreasing on $[c, \infty)$. Moreover, a simple limit argument shows that the hypothesis that $\pi^{*} S\left(x, c ; U_{0}\right) \pi \rightarrow \infty$ as $x \rightarrow \infty$ for arbitrary non-null constant vectors $\pi$ is equivalent to the condition that $\rho_{1}(x) \rightarrow \infty$ as $x \rightarrow \infty$, which in turn is equivalent to the condition that $S^{-1}\left(x, c ; U_{0}\right) \rightarrow 0$ as $x \rightarrow \infty$. That is, $\left(U_{0}(x) ; V_{0}(x)\right)$ is a principal solution of the matrix differential equation (3.1), as defined by Reid [20].

THEOREM 3.2. Suppose that hypotheses $\left(H_{1}\right),\left(H_{2}\right),\left(H_{3}\right)$ hold, $K(x)$ is a continuous hermitian matrix satisfying $P_{* *}[c, d]$ for arbitrary $[c, d] \subset X$, while for arbitrary $b \in X$ there exists $a c>b$ such that $\lambda_{n}^{* *}[b, c]<0$. Then for arbitrary $b \in X$ there exists $a d>b$ such that $\lambda_{n}^{* 0}[b, d]<0$, and for $x_{1} \in X$ a necessary and sufficient condition for (2.7) to be nonoscillatory on $\left[x_{1}, \infty\right)$ is that $\lambda_{1}^{0 *}\left[x_{1}, x_{2}\right]>0$ for all $x_{2}>x_{1}$.

If $b \in X$, and $c>b$ is such that $\lambda_{n}^{* *}[b, c]<0$, then by Corollary 1 to Theorem 2.1 there exists an $n \times n$ matrix $G(x)$ whose column vectors belong to $\mathfrak{D}_{* *}[b, c]$ and $\Im[G \pi ; b, c]<0$ for arbitrary nonzero constant vectors $\pi$; that is, $\Im[G \pi ; b, c]$ is a negative definite hermitian form in $\pi$, and hence there exists a positive $\kappa=\kappa[b, c]$ such that $\Im[G \pi ; b, c] \leqq-2 \kappa|\pi|^{2}$ for arbitrary constant vectors $\pi$. Now (3.1) is the Euler-Lagrange canonical matrix differential system for the functional

$$
\Im_{0}[y ; c, d]=\int_{c}^{d} \omega_{0}\left(x, y(x), y^{\prime}(x)\right) d x,
$$

where $\omega_{0}(x, y, \pi) \equiv \pi^{*} R(x) \pi+\pi^{*} Q(x) y+y^{*} Q^{*}(x) \pi+y^{*} P_{0}(x) y$, with $P_{0}(x)$ $=P(x)+C_{0}(x)-C(x)$ and $\Im_{0}[y ; c, d]-\Im[y ; c, d]=\int_{c}^{d} y^{*}\left[C_{0}-C\right] y d x \geqq 0$ for $y(x) \in \mathfrak{D}_{* 0}[c, d]$. For $U_{0}(x), V_{0}(x)$ as in $\left(H_{3}\right)$ and $d>c$, let $U(x)=G(x)$ on $[b, c], U(x)=U_{0}(x)\left[E-S\left(x, c ; U_{0}\right) S^{-1}\left(d, c ; U_{0}\right)\right] G(c)$ on $[c, \infty)$. In particular (see Reid $[20, \S 3])$ on $[c, \infty)$ we have that

$U(x), V(x)=V_{0}(x)\left[E-S\left(x, c ; U_{0}\right) S^{-1}\left(d, c ; U_{0}\right)\right] G(c)-U_{0}^{*-1}(x) S^{-1}\left(d, c ; U_{0}\right) G(c)$

is the solution of (3.1) satisfying $U(c)=G(c), U(d)=0$, and the column vec- 
tors of $U(x)$ are elements of $\mathfrak{D}_{* 0}[b, d]$. Therefore,

$$
\begin{aligned}
\Im[U \pi ; c, d] \leqq \Im_{0}[U \pi ; c, d] & =-\pi^{*} U^{*}(c) V(c) \pi \\
& =-\pi^{*} G^{*}(c)\left[M-S^{-1}\left(d, c ; U_{0}\right)\right] G(c) \pi \\
& \leqq \pi^{*} G^{*}(c) S^{-1}\left(d, c ; U_{0}\right) G(c) \pi,
\end{aligned}
$$

the last inequality resulting from the condition $M \geqq 0$; in view of $\left(H_{3}\right)$ there exists a $d_{0}>c$ such that $\Im[U \pi ; c, d] \leqq \kappa|\pi|^{2}$ for $d \geqq d_{0}$, and consequently $\Im[U \pi ; b, d] \leqq-\kappa|\pi|^{2}$ for such $d$ and arbitrary constant vectors $\pi$. It then follows that the negative index of $\Im[y ; b, d]$ on $\mathfrak{D}_{* 0}[b, d]$ is at least $n$, and thus $\lambda_{n}^{* 0}[b, d]<0$ by Corollary 1 of Theorem 2.1.

Corollary 1. Suppose $C(x) \leqq 0$ on $X$, and for $Y(x)$ a fundamental matrix solution of the first order matrix differential equation

$$
Y^{\prime}=A(x) Y
$$

we have: (i) if $b \in X$ there exists $a c>b$ such that the nonpositive hermitian matrix $\int_{0}^{6} Y^{*}(x) C(x) Y(x) d x$ is negative definite; (ii) if $c \in X$ then

$$
\pi^{*}\left[\int_{0}^{x} Y^{-1}(t) B(t) Y^{*-1}(t) d t\right] \pi \rightarrow \infty
$$

as $x \rightarrow \infty$ for arbitrary non-null constant vectors $\pi$. Then for a given $x_{1} \in X$ the system (2.7) is nonoscillatory on $\left[x_{1}, \infty\right)$ if and only if $\lambda_{1}^{0 *}\left[x_{1}, x_{2}\right]>0$ for all $x_{2}>x_{1}$.

Since $C(x) \leqq 0$, and condition (ii) holds for a fundamental matrix solution $Y(x)$ of (3.3), hypothesis $\left(H_{3}\right)$ is valid for $C_{0}(x) \equiv 0, M=0, U_{0}(x)=Y(x)$, $V_{0}(x) \equiv 0$. In particular, the column vectors of $Y(x)$ are elements of $\mathfrak{D}_{* *}[b, c]$ for given $[b, c] \subset X$, and for arbitrary constant vectors $\pi$ it may be verified readily that $\omega\left(x, Y(x) \pi, Y^{\prime}(x) \pi\right) \equiv \pi^{*} Y^{*}(x) C(x) Y(x) \pi$. Consequently, if $c$ is a value satisfying condition (i) of the corollary then the negative index of $\Im[y ; b, c]$ on $\mathfrak{D}_{* *}[b, c]$ is at least $n$, so that $\lambda_{n}^{* *}[b, c]<0$ by Corollary 1 to Theorem 2.1, and the conclusion of the corollary is an immediate consequence of Theorem 3.2.

If in addition to the conditions of Corollary 1 the matrix of (i) is such that $\pi^{*}\left[\int_{0}^{c} Y^{*}(x) C(x) Y(x) d x\right] \pi \rightarrow-\infty$ as $c \rightarrow \infty$ for arbitrary non-null constant vectors $\pi$, then for a given $x_{1} \in X$ it follows that for $x_{2}$ sufficiently large the negative index of $\Im\left[y ; x_{1}, x_{2}\right]$ on $\mathfrak{B}_{0 *}\left[x_{1}, x_{2}\right]$ is at least $n$, so that $\lambda_{n}^{0 *}\left[x_{1}, x_{2}\right]$ $<0$ for $x_{2}$ sufficiently large, and by the above Corollary 1 the system (2.7) is oscillatory on $\left[x_{1}, \infty\right)$. However, an independent but related argument establishes the following stronger result.

TheOREM 3.3. Suppose $C(x) \leqq 0$ on $X$, and for $Y(x)$ a fundamental matrix solution of (3.3) we have condition (ii) of Corollary 1 , and ( $\left.\mathrm{i}^{\prime}\right)$ : there exists a 
non-null constant vector $\pi_{0}$ such that $\pi_{0}^{*}\left[\int_{b}^{c} Y^{*}(x) C(x) Y(x) d x\right] \pi_{0} \rightarrow-\infty$ as $c \rightarrow \infty$. Then for a given $x_{1} \in X$ the equation (2.7) is oscillatory on $\left[x_{1}, \infty\right)$.

For $x_{1} \in X, b>x_{1}$, and $\pi_{0}$ a constant vector satisfying $\left(i^{\prime}\right)$, let $y_{0}(x)$ be an element of $\mathfrak{D}_{0 *}\left[x_{1}, b\right]$ such that $y_{0}(b)=Y(b) \pi_{0}$. Such a $y_{0}(x)$ may be chosen in many ways; for example, if $\lambda_{0}$ is not a proper value of $B_{00}\left[x_{1}, b\right]$ then for $\lambda=\lambda_{0}$ there is a unique solution $u(x), v(x)$ of (2.9) with $u\left(x_{1}\right)=0, u(b)=Y(b) \pi_{0}$, and $y_{0}(x)$ may be chosen as $u(x)$. By condition ( $\left.i^{\prime}\right)$ there exists a $c>b$ such that $\pi_{0}^{*}\left[\int_{b}^{c} Y^{*}(x) C(x) Y(x) d x\right] \pi_{0} \leqq-\Im\left[y_{0} ; x_{1}, b\right]-2\left|\pi_{0}\right|^{2}$. Moreover, the proof of Theorem 3.2 for $U_{0}(x)=Y(x), V_{0}(x) \equiv 0$ shows that if $d>c$ and $(U(x) ; V(x))$ is the solution of $U^{\prime}=A(x) U+B(x) V, V^{\prime}=-A^{*}(x) V$ satisfying $U(c)=Y(c)$, $U(d)=0$, then $\Im[U \pi ; c, d] \rightarrow 0$ as $d \rightarrow \infty$ for arbitrary constant vectors $\pi$. In particular, if $d=x_{2}$ is a value such that $\Im\left[U \pi_{0} ; c, x_{2}\right] \leqq\left|\pi_{0}\right|^{2}$, then $\Im\left[y ; x_{1}, x_{2}\right]$ $<0$ for the $y(x) \in \mathfrak{D}_{00}\left[x_{1}, x_{2}\right]$ defined as: $y(x)=y_{0}(x)$ on $\left[x_{1}, b\right], y(x)=Y(x) \pi_{0}$ on $[b, c], y(x)=U(x) \pi_{0}$ on $\left[c, x_{2}\right]$. Consequently, for such a value $x_{2}$ the negative index of $\Im\left[y ; x_{1}, x_{2}\right]$ on $\mathfrak{D}_{00}\left[x_{1}, x_{2}\right]$ is at least one, so that $(2.7)$ is oscillatory on $\left[x_{1}, x_{2}\right]$.

4. Self-adjoint scalar quasi-differential equations of even order. An important instance of (2.7) is provided by certain systems which are equivalent to self-adjoint scalar quasi-differential equations of even order. Suppose $p_{j}(x)$, $(j=0,1, \cdots, 2 n)$, are real-valued continuous functions with $p_{2 n}(x)>0$ on $X: a<x<\infty$, and let $R(x), Q(x), P(x)$ be diagonal matrices with $P_{\alpha \alpha}(x)$ $=p_{2 \alpha-2}(x), Q_{\alpha \alpha}(x)=i p_{2 \alpha-1}(x)$ for $\alpha=1, \cdots, n$, while $R_{\beta \beta}(x) \equiv 0$ for $\beta=1, \cdots$, $n-1, R_{n n}(x)=p_{2 n}(x)$, and $\Phi(x, y, \pi)=\left(\pi_{\beta}-y_{\beta+1}\right),(\beta=1, \cdots, n-1)$. The corresponding system (2.7) is then

$$
\begin{array}{rlr}
u_{\alpha}^{\prime} & =u_{\alpha+1} & (\alpha=1, \cdots, n-1), \\
u_{n}^{\prime} & =-i\left(p_{2 n-1} / p_{2 n}\right) u_{n}+\left(1 / p_{2 n}\right) v_{n}, & \\
v_{1}^{\prime} & =p_{0} u_{1}-i p_{1} u_{2}, & \\
v_{\alpha}^{\prime} & =i p_{2 \alpha-3} u_{\alpha-1}+p_{2 \alpha-2} u_{\alpha}-i p_{2 \alpha-1} u_{\alpha+1}-v_{\alpha-1} & (\alpha=2, \cdots, n-1), \\
v_{n}^{\prime} & =i p_{2 n-8} u_{n-1}+\left(p_{2 n-2}-p_{2 n-1}^{2} / p_{2 n}\right) u_{n}-v_{n-1}-i\left(p_{2 n-1} / p_{2 n}\right) v_{n},
\end{array}
$$

and it follows readily that this system satisfies hypotheses $\left(H_{1}\right)$ and $\left(H_{2}\right)$. Moreover, $(u ; v)$ is a solution of the vector system (4.1) if and only if there exists a scalar function $w(x) \in C^{(n)}$ and associated $v_{j}(x) \in C^{\prime},(j=1, \cdots, n)$, such that

$$
\begin{aligned}
& u_{j}=w^{(j-1)} \\
& v_{n}=p_{2 n} w^{(n)}+i p_{2 n-1} w^{(n-1)} \text {, } \\
& v_{1}^{\prime}=p_{0} w-i p_{1} w^{\prime}, \\
& v_{\beta+1}^{\prime}=i p_{2 \beta-1} w^{(\beta-1)}+p_{2, q} w^{(\beta)}-i p_{2 \beta+1} w^{(\beta+1)}-v_{\beta} \quad(\beta=1, \cdots, n-1) \text {. } \\
& (j=1, \cdots, n),
\end{aligned}
$$


If $w, v_{1}, \cdots, v_{n}$ is a solution of (4.2), then in general the function $w$ does not have derivatives of order higher than $n$ and (4.2) is not equivalent to a linear differential equation of the form $\sum_{j=1}^{2 n} l_{j}(x) w^{(i)}$; however, (4.2) is equivalent to the quasi-differential equation (see Bocher [5]; Achieser-Glasmann [1, Anhang II]; Reid [19, p. 451]),

$$
D^{(2 n)} w=0,
$$

where for $n=1,2, \ldots$ and a function $w(x) \in C^{(n)}$ we use the following modification of the formal derivative operator $D$, as determined by the set of coefficient functions $p_{j}(x)$,

$$
\begin{array}{rlr}
D^{(k)} & =D^{k} \quad \quad(k=0,1, \cdots, n-1) ; \\
D^{(n)} & =p_{2 n} D^{n}+i p_{2 n-1} D^{n-1} ; \\
D^{(n+j)} & =D D^{\langle n+j-1\rangle}-(-1)^{j}\left[i p_{2 n-2 j+1} D^{n-i+1}-p_{2 n-2 j} D^{n-j}\right. \\
& \left.-i p_{2 n-2 j-1} D^{n-j-1}\right] \quad(j=1, \cdots, n-1) ; \\
D^{(2 n)} & =D D^{(2 n-1)}-(-1)^{n}\left[i p_{1} D-p_{0} D^{0}\right] .
\end{array}
$$

For example, for $n=1$ and $n=2$ equation (4.3) becomes, respectively,

$$
\begin{aligned}
\left(p_{2} w^{\prime}+i p_{1} w\right)^{\prime}+i p_{1} w^{\prime}-p_{0} w & =0, \\
\left(\left[p_{4} w^{\prime \prime}+i p_{3} w^{\prime}\right]^{\prime}+i p_{3} w^{\prime \prime}-p_{2} w^{\prime}-i p_{1} w\right)^{\prime}-i p_{1} w^{\prime}+p_{0} w & =0 .
\end{aligned}
$$

If for $j=0,1, \cdots, 2 n$ we have that $p_{j}(x)$ is of class $C^{(j / 2)}$ or $C^{((j+1) / 2)}$ according as $j$ is even or odd, then (4.2) is satisfied by a set $w, v_{1}, \cdots, v_{n}$ if and only if $w \in C^{(2 n)}$, and in this case (4.3) may be written

$$
\begin{aligned}
\sum_{j=0}^{n}(-1)^{n-j}\left[p_{2 j} w^{(j)}\right]^{(j)}-i \sum_{k=0}^{n-1}(-1)^{n-k} & \left(\left[p_{2 k+1} w^{(k)}\right]^{(k+1)}\right. \\
& \left.+\left[p_{2 k+1} w^{(k+1)}\right]^{(k)}\right)=0 ;
\end{aligned}
$$

that is, (4.3) is of the form of the general self-adjoint scalar differential equation of order $2 n$ (see Reid [19]). Moreover, in this case for $j=1, \cdots, n$,

$$
\begin{aligned}
v_{j}= & \sum_{\alpha=j}^{n}(-1)^{\alpha-i}\left[p_{2 \alpha} w^{(\alpha)}\right]^{(\alpha-j)}+i p_{2 j-1} w^{(j-1)} \\
& +i \sum_{\beta=j}^{n-1}(-1)^{\beta-j-1}\left(\left[p_{2 \beta+1} w^{(\beta+1)}\right]^{(\beta-j)}+\left[p_{2 \beta+1} w^{(\beta)}\right]^{(\beta-j+1)}\right) .
\end{aligned}
$$

Employing the terminology of the preceding sections for the corresponding first order system, a point $x_{2}$ is termed conjugate to $x_{1}$, relative to (4.3), provided there exists a nonidentically vanishing solution $w(x)$ of (4.3) satisfying $w^{(\alpha-1)}\left(x_{1}\right)=w^{(\alpha-1)}\left(x_{2}\right),(\alpha=1, \cdots, n)$. Moreover, we shall say that this equation is nonoscillatory on a subinterval $X_{0}$ of $X$ if no two distinct points of 
$X_{0}$ are mutually conjugate. It is to be emphasized that these definitions of conjugate point and oscillation for (4.3) are the more restrictive ones arising from the corresponding concepts for the associated first order system (4.1) in the sense of $\$ 2$, and do not have the generality corresponding to the definitions introduced by Leighton and Nehari [10] for real fourth order equations

$$
\left(r(x) u^{\prime \prime}\right)^{\prime \prime}-p(x) u=0 .
$$

For (4.3) a definition of conjugate point corresponding to that of Leighton and Nehari [10] would be that a value $x_{2}>x_{1}$ is the $k$ th (right-hand) conjugate point of $x_{1}$ if $x_{2}$ is the minimum value of $a_{k+2 n-1}$ such that there is a nonidentically vanishing solution $w(x)$ of $(4.3)$ with zeros at $x=a_{j},(j=1, \cdots, k+2 n-1)$, where $x_{1}=a_{1} \leqq a_{2} \leqq \cdots \leqq a_{k+2 n-1}$. However, for the case of an equation (4.7) with positive coefficients $r(x) \in C^{\prime \prime}, p(x) \in C$, it follows from Theorem 3.6 of [10] that a value $x_{2}>x_{1}$ is conjugate to $x_{1}$ in one of the above described senses if and only if it is conjugate in the other sense, and consequently application of results of this section to (4.7) does provide a variational proof of Theorem 2.1 of Howard [7]. At present it is not known for what classes of equations (4.3) the two concepts of conjugacy are equivalent, and the present paper makes no contribution to the solution of this problem.

For brevity, special criteria for (4.3) will be limited to cases covered by Corollary 1 of Theorem 3.2, and Theorem 3.3. The solution $Y(x)$ of the corresponding equation (3.3) satisfying $Y(c)=E$ is readily found to have elements $Y_{\alpha \beta}(x)$ as follows: $Y_{\alpha \beta}(x) \equiv 0$ for $\alpha>\beta ; Y_{\alpha \beta}(x)=(x-c)^{\beta-\alpha} /(\beta-\alpha)$ ! for $1 \leqq \alpha \leqq \beta \leqq n-1$; if $r(x)=-p_{2 n-1}(x) / p_{2 n}(x)$, then $Y_{n n}(x)=\exp \left[i \int_{c}^{x} r(s) d s\right]$, and $Y_{\alpha n}(x)=\int_{c}^{x}\left[(x-t)^{n-\alpha-1} /(n-\alpha-1) !\right] Y_{n n}(t) d t$ for $1 \leqq \alpha \leqq n-1$. Moreover, since $W(x)=Y^{*-1}(x)$ is the solution of the matrix differential equation $W^{\prime}=-A^{*}(x) W$ satisfying $W(c)=E$, the elements $W_{\alpha \beta}(x)$ are as follows: $W_{\alpha \beta}(x) \equiv 0$ for $\beta>\alpha ; W_{\alpha \beta}(x)=(-1)^{\alpha-\beta}(x-c)^{\alpha-\beta} /(\alpha-\beta)$ ! for $1 \leqq \beta \leqq \alpha \leqq n-1$; $W_{n \beta}(x)=z_{n \beta}(x) \exp \left[i \int_{c}^{z} r(s) d s\right], \quad(\beta=1, \cdots, \quad n), \quad$ where $z_{n n}(x) \equiv 1, \quad z_{n \beta}(x)$ $=\left[(-1)^{n-\beta} /(n-\beta-1) !\right] \int_{c}^{x}(t-c)^{n-\beta-1} \exp \left[-i \int_{c}^{t} r(s) d s\right] d t,(\beta=1, \cdots, n-1)$.

Of particular interest is the case in which $p_{2 n-1}(x) \equiv 0$, since in this instance $Y_{\alpha \beta}(x) \equiv 0$ for $\alpha>\beta, Y_{\alpha \beta}(x)=(x-c)^{\beta-\alpha /(\beta-\alpha) !}$ for $1 \leqq \alpha \leqq \beta \leqq n$, and $W_{\alpha \beta}(x) \equiv 0$ for $\beta>\alpha, W_{\alpha \beta}(x)=(-1)^{\alpha-\beta}(x-c)^{\alpha-\beta} /(\alpha-\beta)$ ! for $1 \leqq \beta \leqq \alpha \leqq n$. In this case the hermitian form $\pi^{*} C(x) \pi$ is the Jacobi form

$$
J(x ; \pi) \equiv \sum_{\alpha=1}^{n} p_{2 \alpha-2}(x)\left|\pi_{\alpha}\right|^{2}-i \sum_{\alpha=1}^{n-1} p_{2 \alpha-1}(x)\left[\bar{\pi}_{\alpha} \pi_{\alpha+1}-\bar{\pi}_{\alpha+1} \pi_{\alpha}\right] .
$$

Moreover, if $\eta=\eta(x \mid \pi)$ denotes the vector polynomial $\left(\eta_{\alpha}(x \mid \pi)\right)$ with

$$
\eta_{\alpha}(x \mid \pi)=\left[\sum_{\beta=1}^{n} \pi_{\beta}(x-c)^{\beta-1} /(\beta-1) !\right]^{(\alpha-1)} \quad(\alpha=1, \cdots, n),
$$

then 


$$
\begin{gathered}
\pi^{*}\left[\int_{b}^{c} Y^{*}(x) C(x) Y(x) d x\right] \pi=\int_{b}^{c} J(x ; \eta(x \mid \pi)) d x \\
\pi^{*}\left[\int_{c}^{x} Y^{-1}(t) B(t) Y^{*-1}(t) d t\right] \pi \\
=\int_{c}^{x}\left[p_{2 n}(t)\right]^{-1}\left|\sum_{\beta=1}^{n} \frac{(-1)^{n-\beta}}{(n-\beta) !}(t-c)^{n-\beta} \pi_{\beta}\right|^{2} d t .
\end{gathered}
$$

From (4.11) it is seen that condition (ii) of Corollary 1 to Theorem 3.2 is equivalent to the condition that $\int_{0}^{\infty}\left[p_{2 n}(t)\right]^{-1} d t=\infty$. Moreover, $C(x) \leqq 0$ on $X$ and condition (i) of the corollary holds whenever there exists a non-negative continuous $q(x)$ which is not identically zero on any $\left[a_{1}, \infty\right) \subset X$, and is such that

$$
J(x ; \pi)+q(x)\left|\pi_{1}\right|^{2} \leqq 0 \text { for arbitrary } \pi=\left(\pi_{\alpha}\right), \quad(\alpha=1, \cdots, n) .
$$

Consequently, for (4.3) Corollary 1 to Theorem 3.2 provides the following criterion.

TheOREM 4.1. If $p_{2 n-1}(x) \equiv 0, \int_{0}^{\infty}\left[p_{2 n}(t)\right]^{-1} d t=\infty$, and (4.12) holds for a continuous $q(x) \geqq 0$ which is not identically zero on any $\left[a_{1}, \infty\right) \subset X$, then for $a$ given $x_{1} \in X$ the equation (4.3) is nonoscillatory on $\left[x_{1}, \infty\right)$ if and only if for arbitrary $x_{2}>x_{1}$ the functional

$$
\int_{x_{1}}^{x_{2}}\left\{\sum_{\alpha=0}^{n} p_{2 \alpha}(x)\left|\eta^{(\alpha)}\right|^{2}+i \sum_{\beta=1}^{n-1} p_{2 \beta-1}(x)\left[\tilde{\eta}^{(\beta)} \eta^{(\beta-1)}-\bar{\eta}^{(\beta-1)_{\eta}(\beta)}\right]\right\} d x
$$

is positive definite in the class of functions $\eta(x) \in C^{(n-1)}\left[x_{1}, x_{2}\right]$ with $\eta^{(n-1)}(x)$ a.c. and $\eta^{(n)}(x) \in \mathbb{R}_{2}\left[x_{1}, x_{2}\right]$, while $\eta^{(\alpha-1)}\left(x_{1}\right)=0,(\alpha=1, \cdots, n)$.

In view of the results of $\$ 2$, the stated property of positive definiteness of the functional (4.13) may be phrased in terms of the smallest proper value of various associated boundary problems. In particular, if $p_{0}(x)$ does not vanish identically on any nondegenerate subinterval of $X$, then by Theorem 2.3 the stated positive definiteness property of $(4.13)$ is equivalent to the condition that for arbitrary $x_{2}>x_{1}$ the smallest proper value of the boundary problem

$$
D^{2 n)} w+(-1)^{n}(\lambda-1) p_{0}(x) w=0, \quad w^{(\alpha-1)}\left(x_{1}\right)=0=v_{\alpha}\left(x_{2}\right), \quad(\alpha=1, \cdots, n)
$$

be greater than 1 , where the functions $v_{j}(x)$ are defined by (4.2). For $n=1$, $p_{2}(x) \equiv 1, p_{1}(x) \equiv 0$, this criterion reduces to that of Theorem I of Nehari [14]. For $n=2, p_{j}(x) \equiv 0(j=1,2,3)$, the corresponding nonoscillation criterion is that of Theorem 2.1 of Howard [7]. For $n=2, p_{1}(x) \equiv 0 \equiv p_{3}(x)$, the result of Theorem 4.1 is equivalent to the special case of Theorem 3.1 of Barrett [2] 
provided by the corresponding equations $D^{(1)} w=0$ in which $p_{0}(x)$ is not identically zero for large $x$.

Finally, since by a suitable choice of $\pi$ the $\eta_{1}(x \mid \pi)$ of $(4.9)$ is equal to a given polynomial of degree less than $n$, through the choice of $\pi_{0}$ such that $\eta_{1}\left(x \mid \pi_{0}\right)=x^{n-1}$ the following result is a direct consequence of Theorem 3.3.

TheOREM 4.2. If $p_{2 n-1}(x) \equiv 0, \int_{c}^{\infty}\left[p_{2 n}(t)\right]^{-1} d t=\infty$, the form $J(x ; \pi)$ of (4.8) is nonpositive for $x \in X$, and (4.3) is nonoscillatory on $\left[x_{1}, \infty\right)$ for $x_{1}$ sufficiently large, then each of the integrals

$$
\int^{\infty} p_{2 \alpha}(t) t^{2 n-2 \alpha-2} d t, \quad(\alpha=0,1, \cdots, n-1),
$$

is convergent.

For $n=1, p_{1}(x) \equiv 0$, the result of Theorem 4.2 was established by Leighton [9]. For $n=2, p_{1}(x) \equiv 0 \equiv p_{3}(x)$, and $p_{4}(x)$ bounded on $X$, the result is a special case of a criterion established for analogous fourth order matrix differential equations by Sternberg and Sternberg [21]. If $p_{2 n-1}(x) \equiv 0, J(x ; \pi)$ is a nonpositive form for $x \in X$, and one of the integrals (4.15) is divergent, the argument indicated above may be used to prove that for given $c \in X$, and $d$ sufficiently large, the smallest proper value $\lambda_{1}^{0 *}[c, d]$ of the boundary problem $\mathfrak{B}_{0 *}[c, d]$ associated with (4.1) is negative; in particular, this result generalizes Theorem 2.4 of Barrett [2].

\section{BiBLIOGRAPHY}

1. N. I. Achieser and I. M. Glasmann, Theorie der linearen Operatoren im Hilbert-Raum, Akademie-Verlag, Berlin, 1954.

2. J. H. Barrett, Two-point boundary value problems and comparison theorems for fourthorder self-adjoint differential equations and second-order matrix differential equations, Technical Summary Report \#150, April, 1960, Mathematics Research Center, U. S. Army.

3. G. D. Birkhoff and M. R. Hestenes, Natural isoperimetric conditions in the calculus of variations, Duke Math. J. vol. 1 (1935) pp. 198-286.

4. G. A. Bliss, Lectures on the calculus of variations, University of Chicago Press, 1946.

5. M. Bôcher, Applications and generalizations of the concept of adjoint systems, Trans. Amer. Math. Soc. vol. 14 (1913) pp. 403-420.

6. M. R. Hestenes, Applications of the theory of quadratic forms in Hilbert space to the calculus of variations, Pacific J. Math. vol. 1 (1951) pp. 525-581.

7. H. C. Howard, Oscillation criteria for fourth-order linear differential equations, Trans. Amer. Math. Soc. vol. 96 (1960) pp. 296-311.

8. K. S. Hu, The problem of Bolza and its accessory boundary value problem (Dissertation, University of Chicago, 1932), Contributions to the Calculus of Variations, University of Chicago Press, 1931-1932, pp. 361-443.

9. W. Leighton, The detection of the oscillation of solutions of a second order linear differential equation, Duke Math. J. vol. 17 (1950) pp. 57-62.

10. W. Leighton and $Z$. Nehari, On the oscillation of solutions of self-adjoint linear differential equations of the fourth order, Trans. Amer. Math. Soc. vol. 89 (1958) pp. 325-377. 
11. M. Morse, Sufficient conditions in the problem of Lagrange with fixed end points, Ann. of Math. vol. 32 (1931) pp. 567-577.

12. - Sufficient conditions in the problem of Lagrange with variable end conditions, Amer. J. Math. vol. 53 (1931) pp. 517-546.

13. - The calculus of variations in the large, Amer. Math. Soc. Colloquium Publications, vol. 18, 1934.

14. Z. Nehari, Oscillation criteria for second-order linear differential equations, Trans. Amer. Math. Soc. vol. 85 (1957) pp. 428-445.

15. W. T. Reid, A boundary value problem associated with the calculus of variations, Amer. J. Math. vol. 54 (1932) pp. 769-790.

16. - An integro-differential boundary value problem, Amer. J. Math. vol. 60 (1938) pp. 257-292.

17. - A matrix differential equation of Riccati type, Amer. J. Math. vol. 68 (1946) pp. 237-246; also Addendum, ibid., vol. 70 (1948) p. 460.

18. - Oscillation criteria for linear differential systems with complex coefficients, Pacific J. Math. vol. 6 (1956) pp. 733-751.

19. - Adjoint linear differential operators, Trans. Amer. Math. Soc. vol. 85 (1957) pp. 446-461.

20. - Principal solutions of non-oscillatory self-adjoint linear differential equations, Pacific J. Math. vol. 8 (1958) pp. 147-169.

21. H. M. Sternberg and R. L. Sternberg, $A$ two-point boundary problem for ordinary selfadjoint differential equations of fourth order, Canad. J. Math. vol. 6 (1954) pp. 416-419.

STate UNIVERsity OR IOWA, Iowa CrTy, Iowa 